PROCEEDINGS OF THE

AMERICAN MATHEMATICAL SOCIETY

Volume 135, Number 8, August 2007, Pages 2633-2636

S 0002-9939(07)08904-6

Article electronically published on March 14, 2007

\title{
CONSTRUCTION OF NON-ALTERNATING KNOTS
}

\author{
SEBASTIAN BAADER \\ (Communicated by Daniel Ruberman)
}

\begin{abstract}
We investigate the behaviour of Rasmussen's invariant $s$ under the sharp operation on knots and obtain a lower bound for the sharp unknotting number. This bound leads us to an interesting move that transforms arbitrary knots into non-alternating knots.
\end{abstract}

\section{INTRODUCTION}

An unknotting operation is a local operation that allows us to untie every knot in finitely many steps. The most popular unknotting operation is a simple crossing change. Every unknotting operation gives rise to a measure of complexity for knots, called an unknotting number. An effective lower bound for the usual unknotting number was introduced by Rasmussen (4]). His invariant led to an easy computation of the genera and unknotting numbers of torus knots. In this paper, we study the sharp unknotting operation via Rasmussen's invariant $s$.

The sharp unknotting operation is a local move that acts on link diagrams, as shown in Figure 1. It has been introduced by Murakami ([3]) and gives rise to the unknotting number $u_{\#}$. The usual unknotting number is denoted by $u$.

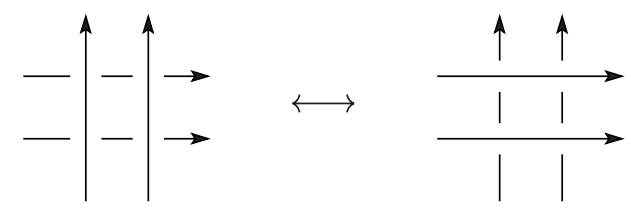

FIGURE 1. Sharp operation

Our main result involves a special sharp operation, called a positive sharp operation. A positive sharp operation introduces eight positive crossings to a link diagram, as shown in Figure 2.

For a diagram $D$ of a knot $K$, we denote by $w(D), O(D)$, and $g(K)$ the writhe of $D$ (i.e., the algebraic crossing number of $D$ ), the number of Seifert circles of $D$, and the genus of $K$, respectively.

Received by the editors March 6, 2006.

2000 Mathematics Subject Classification. Primary 57M27.

(C)2007 American Mathematical Society 


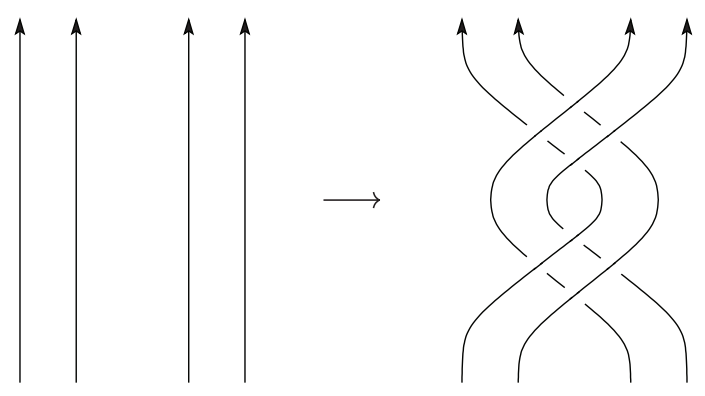

Figure 2. Positive sharp operation

Theorem 1.1. Let $D$ be any knot diagram, and suppose $D^{\prime}$ is obtained from $D$ by the application of $n$ positive sharp operations. If

$$
n>g(K)-\frac{1+w(D)-O(D)}{2},
$$

then $D^{\prime}$ represents a non-alternating knot.

The quantity on the right-hand side of the inequality in Theorem 1.1 is always positive, as follows from Bennequin's inequality ([1]). For positive knot diagrams, it is actually zero.

Corollary 1.2. Let $D$ be a positive knot diagram, and suppose $D^{\prime}$ is obtained from $D$ by the application of one positive sharp operation. Then $D^{\prime}$ represents a non-alternating knot.

\section{Examples 1.3.}

(i) The closure of the braid $\sigma_{1}^{-1} \sigma_{2} \sigma_{1} \sigma_{3} \sigma_{2}$ represents the trivial knot $O$. For the corresponding knot diagram $D$ (see Figure 3, on the left-hand side), Bennequin's inequality is an equality:

$$
g(O)-\frac{1}{2}(1+w(D)-O(D))=0-\frac{1}{2}(1+3-4)=0 .
$$

Therefore, if we apply one positive sharp operation at the top of this braid diagram, we obtain a non-alternating knot. It is a 2-cable of the positive trefoil knot.

(ii) The closure of the braid $\sigma_{1} \sigma_{2} \sigma_{3}$ represents the trivial knot, too (see Figure 3 , on the right-hand side). By Corollary 1.2, the application of one positive sharp operation at the top of that braid diagram yields a nonalternating knot. This time, we obtain the knot $10_{139}$, in Rolfsen's notation ([5]).

A sharp unknotting operation changes 4 crossings of a diagram. Therefore, $u$ cannot exceed $4 u_{\#}$. Rasmussen's invariant allows us to detect knots with $u(K)=$ $4 u_{\#}(K)$.

\section{Theorem 1.4.}

(1) $u_{\#}(K) \geqslant \frac{|s(K)|}{8}$.

(2) If $u_{\#}(K)=\frac{|s(K)|}{8}$, then $K$ is either trivial or non-alternating. In any case, $u(K)=4 u_{\#}(K)$ holds. 

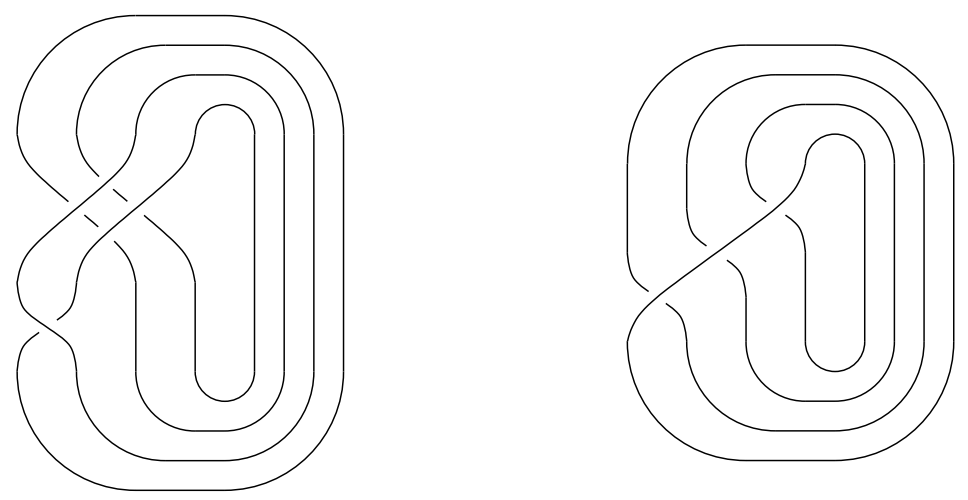

Figure 3. Two diagrams of the trivial knot

Examples 1.5. (i) The diagram $D$ of the 2-cable knot $K$ we constructed above has 4 Seifert circles and writhe 11 , whence $1+w(D)-O(D)=8$. The latter quantity is a lower bound for the invariant $s(K)$ (see [7). This proves $u_{\#}(K)=\frac{|s(K)|}{8}=1$.

(ii) The diagram $D$ of the knot $10_{139}$ we constructed above has 4 Seifert circles and writhe 11. Again, we conclude $u_{\#}\left(10_{139}\right)=\frac{\left|s\left(10_{139}\right)\right|}{8}=1$.

\section{RASMUSSEN'S INVARIANT AND THE SHARP UNKNOTTING OPERATION}

The proofs of Theorems 1.1 and 1.4 are based upon the following three properties of Rasmussen's invariant $s$ :

(1) $|s(K)| \leqslant 2 u(K)$,

(2) $s(K)=\sigma(K)$, for all alternating knots $K$ (here $\sigma(K)$ is the signature of the $\operatorname{knot} K)$,

(3) $1+w(D)-O(D) \leqslant s(K)$, where $D$ is any diagram of a knot $K$.

The first two properties were proved by Rasmussen (4]), whereas the third inequality was proved by Shumakovitch ([7]). The main argument in the proof of (3) is Rudolph's reduction to the case of positive diagrams ([6]).

As we remarked after Theorem 1.4, the usual unknotting number $u$ cannot exceed $4 u_{\#}$. Together with the inequality (1), this immediately proves the first statement of Theorem 1.4:

$$
u_{\#}(K) \geqslant \frac{u(K)}{4} \geqslant \frac{|s(K)|}{8} .
$$

In [3], Murakami proved the following estimate for $u_{\#}$, in terms of the signature $\sigma$ of a knot:

$$
u_{\#}(K) \geqslant \frac{|\sigma(K)|}{6} .
$$

This implies the second statement of Theorem 1.4: 
Let $K$ be a knot with $u_{\#}(K)=\frac{|s(K)|}{8}$. Murakami's inequality tells us that

$$
\frac{|s(K)|}{8} \geqslant \frac{|\sigma(K)|}{6} \text {. }
$$

If, in addition, $K$ is alternating, then $s(K)=\sigma(K)$, by (2). Therefore, $s(K)=$ $\sigma(K)=0, u_{\#}(K)=0$, and $K$ is the trivial knot. In any case, $4 u_{\#}(K)=u(K)$ holds.

In order to prove Theorem 1.1, we have to study the behaviour of the numbers $w(D)$ and $O(D)$ under a positive sharp operation: a positive sharp operation increases the writhe by 8 and leaves the number of Seifert circles invariant. Now, let $D$ be any knot diagram of a knot $K$. Furthermore, suppose $D^{\prime}$ is obtained from $D$ by the application of $n$ positive sharp operations. $D^{\prime}$ represents a knot $K^{\prime}$. Using (3), we find the following lower bound for $s\left(K^{\prime}\right)$ :

$$
s\left(K^{\prime}\right) \geqslant 1+w\left(D^{\prime}\right)-O\left(D^{\prime}\right)=1+w(D)+8 n-O(D) .
$$

On the other hand, we have the following upper bound for the signature $\sigma\left(K^{\prime}\right)$ :

$$
\sigma\left(K^{\prime}\right) \leqslant \sigma(K)+6 n \leqslant 2 g(K)+6 n .
$$

The first inequality is due to Murakami (3]): the signature of a knot cannot increase by more than 6 under a sharp operation. The second inequality is obvious, since the signature of a knot $K$ is the signature of a Seifert matrix of size $2 g(K)$. Now, if

then

$$
n>g(K)-\frac{1+w(D)-O(D)}{2},
$$

$$
s\left(K^{\prime}\right)-\sigma\left(K^{\prime}\right) \geqslant 2 n-2 g(K)+1+w(D)-O(D)>0,
$$

whence $K^{\prime}$ is non-alternating. This completes the proof of Theorem 1.1.

Remark 2.1. Throughout this paper, we could replace Rasmussen's invariant $s$ by the concordance invariant $2 \tau$ coming from knot Floer homology, since the three properties (1), (2) and (3) are also valid for $2 \tau$. A list of properties that are shared by $s$ and $2 \tau$ is contained in [2. In the same paper, M. Hedden and P. Ording show that the invariants $s$ and $2 \tau$ are not equal.

\section{REFERENCES}

1. D. Bennequin, Entrelacements et équations de Pfaff, Astérisque 107-108 (1982), 87-161. MR753131 (86e:58070)

2. M. Hedden; Ph. Ording, The Ozsváth-Szabó and Rasmussen concordance invariants are not equal, arXiv: math.GT/0512348, 2005.

3. H. Murakami, Some metrics on classical knots, Math. Ann. 270 (1985), no.1, 35-45. MR769605 (86g:57007)

4. J. Rasmussen, Khovanov homology and the slice genus, arXiv: math.GT/0402131, 2004.

5. D. Rolfsen, Knots and Links, Publish or Perish (1976). MR0515288 (58:24236)

6. L. Rudolph, Quasipositivity as an obstruction to sliceness, Bull. Amer. Math. Soc. 29 (1993), no.1, 51-59. MR1193540 (94d:57028)

7. A. Shumakovitch, Rasmussen invariant, slice-Bennequin inequality, and sliceness of knots, arXiv: math.GT/0411643, 2004.

Mathematisches Institut, ETH ZÜRICh, RÄMistrasse 101, 8092 Zürich, SwitZerland

E-mail address: sebastian.baader@math.ethz.ch 